\title{
Log canonical thresholds on group compactifications
}

\author{
Thibaut Delcroix
}

\begin{abstract}
We compute the log canonical thresholds of non-negatively curved singular hermitian metrics on ample linearized line bundles on bi-equivariant group compactifications of complex reductive groups. To this end, we associate with any such metric a convex function whose asymptotic behavior determines the log canonical threshold. As a consequence we obtain a formula for the alpha invariant of these line bundles, in terms of the polytope associated with the group compactification.
\end{abstract}

\section{Introduction}

The aim of this article is to begin the study of Kähler metrics on polarized $G \times G$-equivariant compactifications of a connected complex reductive group $G$. This class of manifolds generalizes the well-known class of polarized toric manifolds, and we extend some techniques of toric geometry to this setting. In this article, we study singular hermitian metrics on linearized ample line bundles, which are non-negatively curved and invariant under the action of $K \times K$, where $K$ is a maximal compact subgroup of $G$. We associate with such a metric a convex function on some real vector space that we call the convex potential of the metric, and show how the asymptotic behavior of this function is controlled by a polytope associated with the line bundle. This generalizes the case of toric manifolds and relies on the $K A K$-decomposition of a reductive group.

The correspondence between metrics and their convex potentials is a bijection and provides a description of the set of non-negatively curved, $K \times K$-invariant, singular hermitian metrics. Furthermore, to obtain this description we use a special continuous reference metric that generalizes the Batyrev-Tschinkel metric on toric line bundles, which has already been used as a model for the behavior of continuous metrics in [CLT10]. Let us mention here that metrics on compactifications of semisimple groups were also studied in [STBT07] in the adelic context.

We then proceed to compute the log canonical threshold of such metrics, in terms of the asymptotic behavior of their convex potential. To achieve that goal, we associate with the metric a convex body that we call the Newton body of the metric, which gives another way to encode the asymptotic behavior of the convex potential and is well suited to fan decompositions. We should stress at this point that another important ingredient is the existence, in any group compactification, of a toric subvariety (and corresponding fan) that contains the information about the compactification. We obtain the following theorem.

Received 27 October 2015, accepted in final form 12 May 2016.

2010 Mathematics Subject Classification 14M27 (primary), 32Q20 (secondary).

Keywords: $\log$ canonical threshold, group compactification, alpha invariant, convex potential.

This journal is (C) Foundation Compositio Mathematica 2017. This article is distributed with Open Access under the terms of the Creative Commons Attribution Non-Commercial License, which permits non-commercial reuse, distribution, and reproduction in any medium, provided that the original work is properly cited. For commercial re-use, please contact the Foundation Compositio Mathematica. 


\section{T. DELCROIX}

Theorem 1.1. Let $(X, L)$ be a polarized $G \times G$-equivariant compactification of $G$. Assume furthermore that $X$ is Fano. Denote by $P$ the polytope associated with $L$ and by $Q$ the polytope associated with the anticanonical line bundle $-K_{X}$. Let also $H$ denote the convex hull of the images, by the Weyl group $W$ of $G$ of the sum of the positive roots of $G$. Let $h$ be a $K \times K$-invariant hermitian metric with non-negative curvature on $L$; then the log canonical threshold of $h$ is given by

$$
\operatorname{lct}(h)=\sup \{c>0 ; 2 H+2 c P \subset c N(h)+2 Q\},
$$

where $N(h)$ is the Newton body of $h$.

Using this expression of the log canonical threshold, we are able to compute Tian's $\alpha$-invariant for any ample linearized line bundle on a Fano group compactification, with respect to the $K \times K$ action.

Theorem 1.2. Let $(X, L)$ be a polarized compactification of $G$, and let $P:=P(X, L)$. Assume furthermore that $X$ is Fano, and let $Q:=P\left(X,-K_{X}\right)$. Then

$$
\alpha_{K \times K}(L)=\sup \left\{c>0 ; c\left(P+\left(-P^{W}\right)\right) \subset Q \ominus H\right\},
$$

where $P^{W}$ denotes the subset of $W$-invariant points of $P$ and $W$ is the Weyl group of $G$.

This formula generalizes the formula for the $\alpha$-invariant of polarized Fano toric manifolds previously obtained in [Del15a] and independently by other authors [Amb14, LSY15]. In the case of the anticanonical line bundle on a Fano toric manifold, the formula was initially obtained by Song [Son05].

The original motivation for this work was to obtain such an expression, hoping that Tian's criterion for the existence of Kähler-Einstein metrics in terms of this invariant would be satisfied by some group compactifications. Recall that Tian's criterion [Tia87] is that if the $\alpha$-invariant is strictly greater than $n /(n+1)$, where $n$ is the dimension of the manifold, then there exists a Kähler-Einstein metric.

For toric manifolds, additional symmetries had to be taken into account for the criterion to be satisfied. For the important examples of wonderful compactifications of semisimple adjoint groups with no rank one factor, Brion computed their automorphism groups in [Bri07], so that our result allows us to compute the alpha invariant with respect to a maximal compact subgroup of the automorphism group, but Tian's criterion is not satisfied. Even though we can in some cases consider additional symmetries, we do not obtain new examples of Kähler-Einstein metrics by this method. Our study of hermitian metrics will be used in [Del17], where we obtain a necessary and sufficient condition for the existence of Kähler-Einstein metrics on a group compactification in terms of the polytope. The present article and [Del17] contain the main results of the author's Ph.D. thesis [Del15b].

\section{Group compactifications}

\subsection{Definition and examples}

Let $G$ be a connected complex reductive group.

Definition 2.1. Let $X$ be a projective manifold. We say that $X$ is a smooth $G \times G$-equivariant compactification of $G$ (or for short a compactification of $G$ ) if $X$ admits a holomorphic $G \times G$ action with an open and dense orbit equivariantly isomorphic to $G$ as a $G \times G$-homogeneous space under the action defined by $\left(g_{1}, g_{2}\right) \cdot g=g_{1} g g_{2}^{-1}$. 


\section{LOG CANONICAL THRESHOLDS ON GROUP COMPACTIFICATIONS}

Let $X$ be a compactification of $G$. We will always identify $G$ with the open and dense orbit in $X$. These manifolds belong to the class of spherical manifolds [Per14, Tim11]. There is a finite number of $G \times G$-orbits in $X$, and the boundary $X \backslash G$ is of codimension one.

ExAmple 2.2. If $G=T \simeq\left(\mathbb{C}^{*}\right)^{n}$ is a torus, then the compactifications of $T$ are the projective toric manifolds. One goes from the $T$-action to the $T \times T$-action through the morphism $T \times T \rightarrow T$, $\left(t_{1}, t_{2}\right) \mapsto t_{1} t_{2}^{-1}$.

Example 2.3. Assume that $G$ is an adjoint semisimple group. Then De Concini and Procesi [DCP83] showed the existence of a special compactification of $G$, called the wonderful compactification of $G$. It is the only compactification of $G$ satisfying the following property: its boundary $X \backslash G$ (where we identify the open and dense orbit in $X$ with $G$ ) is a union of simple normal crossing prime divisors $D_{i}$ for $i \in\{1, \ldots, r\}$ such that for any subset $I \subset\{1, \ldots, r\}$, the intersection $X \cap \bigcap_{i \in I} D_{i}$ is the closure of a unique $G \times G$-orbit, and all $G \times G$-orbits appear this way. The integer $r$ is equal to the rank of $G$, which is the dimension of a maximal torus in $G$.

The wonderful compactification of $\mathrm{PGL}_{2}(\mathbb{C})$ is especially simple: it is $\mathbb{P}^{3}$ considered as $\mathbb{P}\left(\operatorname{Mat}_{2,2}(\mathbb{C})\right)$ equipped with the action of $\mathrm{PGL}_{2}(\mathbb{C}) \times \mathrm{PGL}_{2}(\mathbb{C})$ induced by the multiplication of matrices on the left and on the right.

\subsection{Polytopes associated with a polarized group compactification}

Recall that a $G$-linearized line bundle over a $G$-manifold $X$ is a line bundle $L$ on $X$ equipped with an action of $G$ lifting the action on $X$ and such that the morphisms between the fibers induced by this action are linear.

Let $G$ be a connected complex reductive group. A polarized group compactification is a pair $(X, L)$, where $X$ is a compactification of $G$ and $L$ is an ample $G \times G$-linearized line bundle on $X$.

Choose a maximal torus $T$ in $G$, and let $K$ be a maximal compact subgroup in $G$. We denote by $M$ the lattice of characters of $T$ and by $N$ the lattice of one-parameter subgroups of $T$. These lattices are naturally dual to each other. Denote by $\mathfrak{a}$ the Lie algebra $\operatorname{Lie}(T) \cap i \operatorname{Lie}(K)$. We identify $\mathfrak{a}$ with $N \otimes \mathbb{R}$ and its dual $\mathfrak{a}^{*}$ with $M \otimes \mathbb{R}$.

Let $\Phi \subset \mathfrak{a}^{*}$ denote the root system of $(G, T)$. Let $W$ be its Weyl group. Choose a system of positive roots $\Phi^{+}$. It defines positive Weyl chambers $\mathfrak{a}^{+}$in $\mathfrak{a}$ and $\mathfrak{a}_{+}^{*}$ in $\mathfrak{a}^{*}$.

TheOrem 2.4 ([AB04, Section 2]). Let $(X, L)$ be a polarized group compactification of $G$. Denote by $Z$ the closure of $T$ in $X$. Then $Z$ is a toric manifold, equipped with a $W$-action, and $\left.L\right|_{Z}$ is a $W$-linearized ample toric line bundle on $Z$.

We denote by $P(X, L)$, or $P$ for simplicity, the polytope associated with the ample toric line bundle $\left.L\right|_{Z}$ by the theory of toric varieties [Ful93, Oda88]. The polytope $P$ is a lattice polytope in $M \otimes \mathbb{R}$, and it is $W$-invariant. Define $P^{+}(X, L)=P(X, L) \cap \mathfrak{a}_{+}^{*}$. It is a polytope in $\mathfrak{a}^{*}$, and $P(X, L)$ is the union of the images of $P^{+}(X, L)$ by $W$.

The polytope $P^{+}(X, L)$ encodes the structure of $G \times G$-representation of the space of holomorphic sections of $L$, generalizing the same property for toric line bundles.

Proposition 2.5 ([AB04, Section 2.2]). Let $(X, L)$ be a polarized group compactification; then

$$
H^{0}(X, L) \simeq \bigoplus\left\{\operatorname{End}\left(V_{\alpha}\right) ; \alpha \in M \cap P^{+}(X, L)\right\},
$$

where $V_{\alpha}$ is an irreducible representation of $G$ with highest weight $\alpha$. 


\section{T. DELCROIX}

EXAmple 2.6 ([BK05, Proposition 6.1.11]). The wonderful compactification $X$ of an adjoint semisimple group is Fano. The corresponding polytope $P\left(X,-K_{X}\right)$ is the convex hull of the images by the Weyl group $W$ of the weight $2 \rho+\sum_{i=1}^{r} \alpha_{i}$, where the $\alpha_{i}$ are the simple roots of $\Phi^{+}$ and $2 \rho$ is the sum of the positive roots.

\section{The convex potential}

In this section, we introduce the convex potential of a $K \times K$-invariant, non-negatively curved singular hermitian metric on a polarized group compactification. This correspondence gives a bijection between the set of these metrics and the set of the $W$-invariant convex functions on $\mathfrak{a}$ which satisfy asymptotic behavior conditions.

\subsection{Singular hermitian metrics and potentials}

Let $X$ be a compactification of $G$ and $L$ a linearized ample line bundle on $X$. Given a hermitian metric $h$ on $L$ and a local trivialization $s$ of $L$ on an open subset $U \subset X$, the local potential of $h$ with respect to $s$ is the function $\phi$ defined on $U$ by

$$
\phi(x)=-\ln \left(|s(x)|_{h}^{2}\right) .
$$

We consider here singular hermitian metrics and only require that the potential with respect to any local trivialization be locally integrable. The value $+\infty$ for the potentials is allowed.

We say that a hermitian metric is locally bounded if its potentials with respect to any trivialization on a sufficiently small open subset are bounded. A hermitian metric is smooth (respectively, continuous) if and only if its potentials with respect to any local trivialization are. A continuous hermitian metric is locally bounded.

Given a reference metric $h_{0}$, we define the global potential $\psi$ of $h$ with respect to $h_{0}$ by

$$
\psi(x)=-\ln \left(\frac{|y|_{h}^{2}}{|y|_{h_{0}}^{2}}\right)
$$

for any element $y$ of the fiber $L_{x}$. This is a function on $X$ that can a priori take the values $\pm \infty$. If $s$ is a local trivialization on $U$ and $\phi$ and $\phi_{0}$ are the respective potentials of $h$ and $h_{0}$ with respect to $s$, then $\psi=\phi-\phi_{0}$ on $U$.

\subsection{The convex potential}

Let $(X, L)$ be a polarized compactification of $G$.

We identify $G$ with the open dense orbit in $X$ and first build a trivialization of $L$ on $G$. Choose a non-zero element $1_{e}$ of the fiber $L_{e}$ above the neutral element $e$ of $G$. Define the section $s$ of $L$ on $G$ by $s(g)=(g, e) \cdot 1_{e}$. This section is a trivialization over $G$, equivariant under the action of $G \times\{e\}$, and any such trivialization is a scalar multiple of $s$.

Denote by $\phi$ the local potential of $h$ on $G$ with respect to $s$.

We are interested in hermitian metrics that are invariant under the action of $K \times K$, where $K$ is a maximal compact subgroup of $G$. We will use the classical $K A K$-decomposition of a complex reductive group.

Proposition 3.1 ([Kna02, Theorem 7.39]). Any element $g \in G$ can be written in the form $g=k_{1} \exp (x) k_{2}$, where $k_{1}, k_{2} \in K$ and $x \in \mathfrak{a}^{+}$. Furthermore, $x$ is uniquely determined by $g$. In other words, the set $A^{+}:=\left\{\exp (x) ; x \in \mathfrak{a}^{+}\right\}$is a fundamental domain for the $K \times K$-action on $G$. 


\section{LOG CANONICAL THRESHOLDS ON GROUP COMPACTIFICATIONS}

We first remark that if $h$ is $K \times K$-invariant, then its potential with respect to the section constructed above is still $K \times K$-invariant.

Proposition 3.2. Assume that $h$ is $K \times K$-invariant; then $\phi$ is also $K \times K$-invariant.

Proof. Let $k_{1}, k_{2} \in K$ and $g \in G$. We can first write

$$
\begin{aligned}
s\left(k_{1} g k_{2}\right) & =\left(k_{1} g k_{2}, e\right) \cdot 1_{e} \\
& =\left(k_{1}, k_{2}^{-1}\right)(g, e)\left(k_{2}, k_{2}\right) \cdot 1_{e} .
\end{aligned}
$$

The subgroup $\operatorname{diag}(G)=\{(g, g) \mid g \in G\}$ fixes the neutral element $e \in G$ and thus acts on the fiber $L_{e}$ through a character $\chi$ of $G$, so that $(g, g) \cdot 1_{e}=\chi(g) 1_{e}$.

We can thus compute

$$
\begin{aligned}
\phi\left(k_{1} g k_{2}\right) & =-\ln \left(\left|s\left(k_{1} g k_{2}\right)\right|_{h}^{2}\right) \\
& =-\ln \left(\left|\left(k_{1}, k_{2}^{-1}\right)(g, e)\left(k_{2}, k_{2}\right) \cdot 1_{e}\right|_{h}^{2}\right) ;
\end{aligned}
$$

by the $K \times K$-invariance of $h$, this is

$$
=-\ln \left(\left|(g, e)\left(k_{2}, k_{2}\right) \cdot 1_{e}\right|_{h}^{2}\right)
$$

and, by linearity,

$$
=-\ln \left(\left|\chi\left(k_{2}\right) \|(g, e) \cdot 1_{e}\right|_{h}^{2}\right)
$$

Since $K$ is compact, $\left|\chi\left(k_{2}\right)\right|=1$, so we obtain

$$
\phi\left(k_{1} g k_{2}\right)=-\ln \left(\left|(g, e) \cdot 1_{e}\right|_{h}^{2}\right)=\phi(g) .
$$

Assume that $h$ is in addition non-negatively curved. Then $\phi$ is a $K \times K$-invariant plurisubharmonic function on $G$. Let $u$ be the function on $\mathfrak{a}$ defined by

$$
u(x)=\phi(\exp (x)) .
$$

Then Azad and Loeb proved in [AL92] that the function $u$ is convex and $W$-invariant.

In particular, since we assumed that the local potentials of singular hermitian metrics are locally integrable, the $K \times K$-invariance of $h$ ensures that the functions $u$ and $\phi$ take finite values on $\mathfrak{a}$ and $G$, respectively. Indeed, a convex function that takes an infinite value at a point must take an infinite value on a whole octant starting from that point, and then the corresponding $K \times K$-invariant function on $G$ is not locally integrable.

Definition 3.3. We will call $u$ the convex potential of $h$.

\subsection{The asymptotic behavior of the convex potential}

3.3.1 A special metric. Let us begin by introducing a continuous, $K \times K$-invariant, reference hermitian metric on $L$. We start from the Batyrev-Tschinkel metric defined on toric manifolds, and generalize it to build a refence continuous metric for any polarized group compactification $(X, L)$, with convex potential equal to the support function of the polytope $2 P(X, L)$.

Given a toric manifold $Z$ equipped with a linearized line bundle $D$, there is a natural continuous hermitian metric $h_{D}$ on $D$, invariant under the action of the compact torus, called the Batyrev-Tschinkel metric (see [Mai00, Section 3.3]). If furthermore the line bundle $D$ is ample, then this metric is non-negatively curved and its convex potential is the support function $v$ of the polytope $2 P(Z, D)$. 


\section{T. DELCROIX}

Now, suppose that $(X, L)$ is a polarized group compactification and that $Z$ is the toric submanifold. Denote $\left.L\right|_{Z}$ by $D$. Then $P(Z, D)=P(X, L)$ is $W$-invariant, which implies that the Batyrev-Tschinkel metric $h_{D}$ is $W$-invariant.

We want to extend $h_{D}$ to a continuous $K \times K$-invariant metric $h_{L}$ on $X$. Define $h_{L}$ at $\xi \in L_{g}$ by $|\xi|_{h_{L}}=\left|\left(k_{1}, k_{2}\right) \cdot \xi\right|_{h_{D}}$, for $\left(k_{1}, k_{2}\right) \in K \times K$ such that $k_{1} g k_{2}^{-1} \in T$. We need to check that this is well defined. Since $h_{D}$ is $W$-invariant, we only need to check that, for $t \in T$, if $\left(k_{1}, k_{2}\right) \in \operatorname{Stab}_{K \times K}(t)$, then $|\xi|_{h_{D}}=\left|\left(k_{1}, k_{2}\right) \cdot \xi\right|_{h_{D}}$. But Stab $\operatorname{St}_{\times K}(t)$ acts linearly on the line $L_{t}$, through a character $\chi$. By compactness, $\left|\chi\left(k_{1}, k_{2}\right)\right|=1$, so $\left|\left(k_{1}, k_{2}\right) \cdot \xi\right|_{h_{D}}=\left|\chi\left(k_{1}, k_{2}\right) \xi\right|_{h_{D}}=|\xi|_{h_{D}}$.

\subsubsection{Asymptotic behavior}

ThEOREM 3.4. The singular hermitian $K \times K$-invariant metrics $h$ with non-negative current curvature are in bijection with the convex $W$-invariant functions $u: \mathfrak{a} \longrightarrow \mathbb{R}$ for which there exists a constant $C_{1} \in \mathbb{R}$ such that

$$
u(x) \leqslant v(x)+C_{1}
$$

on $\mathfrak{a}$, where $v$ is the support function of the polytope $2 P(X, L)$. This bijection is obtained by associating with $h$ its convex potential $u$. Furthermore, $h$ is locally bounded if and only if there exists, in addition, a constant $C_{2} \in \mathbb{R}$ such that

$$
v(x)+C_{2} \leqslant \varphi(x) \leqslant v(x)+C_{1} .
$$

Proof. Let $h$ be a singular hermitian $K \times K$-invariant metric with non-negative current curvature on $L$. Let $u$ be its convex potential. Recall that $h_{L}$ denotes the reference continuous metric constructed above, and let $\omega_{L}$ be the curvature current of $h_{L}$. Denote by $\psi$ the potential of $h$ with respect to $h_{L}$ and by $w$ the function on $\mathfrak{a}$ associated with the $K \times K$-invariant function $\left.\psi\right|_{G}$. The function $w$ is equal to $u-v$.

The function $\psi$ is an $\omega_{L}$-plurisubharmonic $\left(\omega_{L}\right.$-psh) function on $X$, so it is in particular bounded from above on $X$. This implies that $w$ is bounded from above. If, furthermore, $h$ is locally bounded, then since $h_{L}$ is also locally bounded, the function $w$ is bounded on $X$. So $w$ is bounded on $\mathfrak{a}$.

Conversely, let $u$ be a convex $W$-invariant function such that $u(x) \leqslant v(x)+C$. We choose any reference metric $h_{0}$ on $L$ that is smooth, positively curved and $K \times K$-invariant. Then by the first direction there exist constants $C_{1}$ and $C_{2}$ such that if $u_{0}$ is the convex potential of $h_{0}$, we have

$$
v(x)+C_{2} \leqslant u_{0}(x) \leqslant v(x)+C_{1} .
$$

Let $\omega_{0}$ be the curvature form of $h_{0}$.

Consider the function $w:=u-u_{0}$. It will be enough to show that the function $\psi$ on $G$ corresponding to $w$ extends to an $\omega_{0}$-psh function on $X$. We denote by $\phi$ and $\phi_{0}$ the $K \times K$ invariant functions defined by $\phi(\exp (x))=u(x)$ and $\phi_{0}(\exp (x))=u_{0}(x)$.

First remark that $\psi=\phi-\phi_{0}$, and by the other direction of the result of Azad and Loeb [AL92], $\phi$ is psh on $G$. The assumption on $u$ implies that $w$, and thus $\psi$, is bounded from above. Indeed, we have

$$
w=u-u_{0} \leqslant v+C-u_{0} \leqslant C-C_{2} .
$$

A classical result on psh functions is that a psh function extends over an analytic subset if and only if it is locally bounded from above. Here, applying that with $\psi$ allows us to extend $\psi$ 


\section{LOG CANONICAL THRESHOLDS ON GROUP COMPACTIFICATIONS}

to an $\omega_{0}$-psh function on $X$. The corresponding singular hermitian metric $h$ has non-negative curvature, is $K \times K$-invariant and has convex potential $u$.

For locally bounded metrics, one just needs to use the refinement that if a psh function is locally bounded, then it extends to a bounded psh function.

\section{Newton bodies}

In this section, we introduce a convex body associated with any non-negatively curved singular $K \times K$-invariant hermitian metric $h$ on an ample linearized line bundle $L$ on a group compactification $X$. We first define a convex set associated with any function which is a natural set to consider in the case of convex functions. Applying this construction to the convex potential of a hermitian metric yields a convex body that is contained in $2 P(X, L)$ and that will be used to compute the log canonical threshold of $h$.

\subsection{The Newton set of a function}

Definition 4.1. Let $f$ be a function $\mathfrak{a} \rightarrow \mathbb{R}$ and $\sigma$ a closed convex cone in $\mathfrak{a}$. The Newton set of $f$ is the following set in $\mathfrak{a}^{*}$ :

$$
N_{\sigma}(f):=\left\{m \in \mathfrak{a}^{*} ; \exists C, \forall x \in \sigma, f(x)-m(x) \geqslant C\right\} .
$$

In the following, we will simply say cone for a closed convex cone. The whole space $\mathfrak{a}$ is a cone, and we will often consider the corresponding Newton set $N_{\mathfrak{a}}(f)$. For any function $f$ and any cone $\sigma$, the Newton set $N_{\sigma}(f)$ is clearly convex.

Recall the definition of the dual cone $\sigma^{\vee}$ of $\sigma$ :

$$
\sigma^{\vee}=\left\{m \in \mathfrak{a}^{*} ; m(x) \geqslant 0 \forall x \in \sigma\right\} .
$$

The Newton set $N_{\sigma}(f)$ is by definition stable under addition of an element of the opposite of the dual cone $\sigma^{\vee} \subset \mathfrak{a}^{*}$. We also write this as $N_{\sigma}(f)=N_{\sigma}(f)+\left(-\sigma^{\vee}\right)$, where the plus sign means the Minkowski sum of sets.

EXAMPLE 4.2. Let $f$ be the affine function $f(x)=m(x)+c$, where $m \in \mathfrak{a}^{*}$ and $c$ is a constant. Then $N_{\sigma}(f)=m+\left(-\sigma^{\vee}\right)$.

Let us record the following elementary properties of Newton sets.

Proposition 4.3. Let $f$ and $g$ be two functions on $\mathfrak{a}$ and $c \in \mathbb{R}$.

- $N_{\sigma}(c f)=c N_{\sigma}(f)$.

- $N_{\sigma}(f+c)=N_{\sigma}(f)$.

- If $f \leqslant g$, then $N_{\sigma}(f) \subset N_{\sigma}(g)$.

- In particular, if for some constants $c_{1}$ and $c_{2}$,

$$
g+c_{1} \leqslant f \leqslant g+c_{2}
$$

on $\sigma$, then $N_{\sigma}(f)=N_{\sigma}(g)$.

- Let $\sigma_{1}$ and $\sigma_{2}$ be two convex cones such that $\sigma=\sigma_{1} \cup \sigma_{2}$; then

$$
N_{\sigma}(f)=N_{\sigma_{1}}(f) \cap N_{\sigma_{2}}(f) .
$$

The last property is very helpful when we want to use a fan decomposition. 


\section{T. Delcroix}

EXAMPLE 4.4. Let $v: \mathfrak{a} \rightarrow \mathbb{R}$ be a piecewise linear function along a finite fan decomposition $\sigma=\cup_{i=1}^{N} \sigma_{i}$, where $N \in \mathbb{N}$ and the $\sigma_{i}$ are convex cones. For $1 \leqslant i \leqslant N$, denote by $v_{i}$ the element of $\mathfrak{a}^{*}$ such that $v(x)=v_{i}(x)$ on $\sigma_{i}$. Then

$$
N_{\sigma}(v)=\bigcap_{i=1}^{N}\left(v_{i}+\left(-\sigma_{i}^{\vee}\right)\right) .
$$

If furthermore $v$ is convex, then $N_{\sigma}(v)=\operatorname{Conv}\left\{v_{i}\right\}+\left(-\sigma^{\vee}\right)$.

\subsection{The Newton set of convex functions}

For this subsection only, we will allow convex functions to take the value $+\infty$. If $f$ is such a function, we define its domain by

$$
\operatorname{dom}(f):=\{x \in \mathfrak{a} ; f(x)<\infty\} .
$$

We impose, however, that all considered functions have a non-empty domain. Outside this subsection, we always assume $\operatorname{dom}(f)=\mathfrak{a}$.

The first remark is that the Newton set of a function $f$ on the whole of $\mathfrak{a}$ is the domain of its Legendre-Fenchel transform (or convex conjugate) $f^{*}$ defined, for $m \in \mathfrak{a}^{*}$, by

$$
f^{*}(m):=\sup \{m(x)-f(x) ; x \in \mathfrak{a}\} .
$$

Let $\sigma$ be a convex cone, and define the convex function $\delta_{\sigma}$ as the indicator function of $\sigma$, that is, $\delta_{\sigma}(x)=0$ if $x \in \sigma$ and $\delta_{\sigma}(x)=\infty$ otherwise. Then it is not hard to check that $N_{\sigma}(f)=N_{\mathfrak{a}}\left(f+\delta_{\sigma}\right)$. In other words, $N_{\sigma}(f)$ is the domain of the convex conjugate of $f+\delta_{\sigma}$.

We will recall a classical result on convex functions which allows us to express the Newton set of a sum as the Minkowski sum of the Newton sets of the summands. First, recall the definition of infimal convolution.

DeFinition 4.5. Let $f$ and $g$ be two convex functions. The infimal convolution of $f$ and $g$ is the function $f \square g$ defined, for $x \in \mathfrak{a}$, by

$$
f \square g(x)=\inf \{f(x-y)+g(y) ; y \in \mathfrak{a}\} .
$$

TheOREM 4.6 ([Roc97, Theorem 16.4]). Let $f$ and $g$ be two convex functions on $\mathfrak{a}$ such that the relative interiors of the domains of $f$ and $g$ have a point in common. Then

$$
(f+g)^{*}(m)=f^{*} \square g^{*} .
$$

Proposition 4.7. Let $\sigma$ be a convex cone and $f$ a convex function with $\operatorname{dom}(f)=\mathfrak{a}$; then

$$
N_{\sigma}(f)=N_{\mathfrak{a}}(f)+\left(-\sigma^{\vee}\right) .
$$

Proof. We have seen that $N_{\sigma}(f)$ is the domain of the convex conjugate of $f+\delta_{\sigma}$, but by Theorem 4.6, this is also the domain of the function $f^{*} \square \delta_{\sigma}^{*}$. We can apply this theorem because the intersection of the domains of $f$ and $\delta_{\sigma}$ is $\sigma$.

The domain of an infimal convolution is the Minkowski sum of the domains of the two functions that are involved, so we just need to compute the domain of $\delta_{\sigma}^{*}$. We can check that, by definition, this is $-\sigma^{\vee}$ and obtain the statement.

Proposition 4.8. Let $f$ and $g$ be two convex functions, both with domain $\mathfrak{a}$, and $\sigma$ a convex cone. Then $N_{\sigma}(f+g)=N_{\sigma}(f)+N_{\sigma}(g)$. 


\section{LOG CANONICAL THRESHOLDS ON GROUP COMPACTIFICATIONS}

Proof. We have, by the previous proposition,

$$
N_{\sigma}(f+g)=N_{\mathfrak{a}}(f+g)+\left(-\sigma^{\vee}\right),
$$

and by the same proof,

$$
N_{\mathfrak{a}}(f+g)=N_{\mathfrak{a}}(f)+N_{\mathfrak{a}}(g)
$$

so

$$
\begin{aligned}
N_{\sigma}(f+g) & =N_{\mathfrak{a}}(f)+N_{\mathfrak{a}}(g)+\left(-\sigma^{\vee}\right) \\
& =N_{\sigma}(f)+N_{\sigma}(g) .
\end{aligned}
$$

\subsection{The Newton body of a metric}

Let $X$ be a compactification of $G$, polarized by $L$. Let $h$ be a $K \times K$-invariant hermitian metric with non-negative curvature on $L$ and $u$ its convex potential with respect to a fixed leftequivariant trivialization of $L$ on $G$, which is a function on $\mathfrak{a}$.

Definition 4.9. We will call the set $N(h):=N_{\mathfrak{a}}(u)$ the Newton body of $h$.

Let $P$ be the polytope corresponding to the polarization $L$.

EXAMPLE 4.10. Let $h_{L}$ be the metric constructed in Section 3.3.1. Its convex potential $v$ is the support function of $2 P$, so $N\left(h_{L}\right)=2 P$, as in Example 4.4. Remark that the convex potential of $h_{L}$ is piecewise linear with respect to the opposite of the fan of the toric subvariety.

Proposition 4.11. The Newton body of $h$ is stable under the action of the Weyl group $W$.

Proof. Let $u$ be the convex potential of $h$, and let $m \in \mathfrak{a}^{*}$. Suppose

$$
u(x)-m(x) \geqslant C
$$

for some constant $C$ and for all $x \in \mathfrak{a}$. Let $w \in W$. By the $W$-invariance of $u$, the inequality is equivalent to

$$
\begin{aligned}
C & \leqslant u(w \cdot x)-m(x) \\
& \leqslant u(w \cdot x)-w^{-1} \cdot m(w \cdot x) .
\end{aligned}
$$

Since $w$ induces a bijection of $\mathfrak{a}$, for all $w \in W$, we have $m \in N(h)$ if and only if $w \cdot m \in N(h)$, which means that $N(h)$ is $W$-invariant.

We can finally translate the information about the asymptotic behavior of metrics in terms of their Newton bodies.

Proposition 4.12. Let $h$ be a $K \times K$-invariant hermitian metric with non-negative curvature on $L$. Then $N(h) \subset 2 P$. If, in addition, $h$ is locally bounded, then $N(h)=2 P$.

Proof. Recall from Section 3.3.1 that the convex potential $u$ of a $K \times K$-invariant hermitian metric $h$ with non-negative curvature on $L$ satisfies

$$
u \leqslant v+C_{2}
$$

on $\mathfrak{a}$ for some constant $C_{2}$, and that if $h$ is locally bounded, then we have in addition

$$
v+C_{1} \leqslant u
$$

for some constant $C_{1}$.

Now the result easily follows from Proposition 4.3 and Example 4.10. 


\section{T. DELCROIX}

\section{Log canonical thresholds}

In this section, we reduce the computation of the log canonical threshold of a $K \times K$-invariant non-negatively curved metric to an integrability problem involving its convex potential, by using the $K A K$-integration formula. We prove an integrability criterion for exponentials of concave functions with respect to the measure $J(x) d x$ appearing in the $K A K$-integration formula, and then use it to obtain an expression of the log canonical threshold in terms of the Newton body of the metric.

\subsection{Log canonical thresholds on compact manifolds}

In this subsection, we first consider a compact complex manifold $X$ that is not necessarily a group compactification and a line bundle $L$ on $X$.

DeFinition 5.1. Let $x$ be a point in $X$ and $h$ a hermitian metric on $L$. The complex singularity exponent (or local log canonical threshold) of $h$ at $x$, which we denote by lct $(h, x)$, is the supremum of all $c>0$ such that $e^{-c \varphi}$ is integrable with respect to Lebesgue measure in a neighborhood of $x$, where $\varphi$ is the potential of $h$ with respect to a trivialization $s$ of $L$ in a neighborhood of $x$.

Remark 5.2. If $h$ is a locally bounded metric, then on a sufficiently small neighborhood of any point, the potential $\varphi$ is a bounded function, so it is integrable. This means that for any such metric, $\operatorname{lct}(h, x)=\infty$ at any point $x$.

Definition 5.3. Let $h$ be a hermitian metric on $L$; then the log canonical threshold of $h$ is defined as

$$
\operatorname{lct}(h)=\inf _{x \in X}(\operatorname{lct}(h, x)) .
$$

Proposition 5.4. Let $h$ be a singular hermitian metric on $L$, let $h_{0}$ be a locally bounded hermitian metric on $L$, and let $\psi$ be the potential of $h$ with respect to $h_{0}$. Moreover, let $d V$ be any smooth volume form on $X$. Then we have

$$
\operatorname{lct}(h)=\sup \left\{c>0 ; \int_{X} e^{-c \psi} d V<\infty\right\} .
$$

We will use this characterization of the log canonical threshold to compute it. The elementary proof is left to the reader. It relies on the fact that if $\varphi$ is a local potential of $h$, then $e^{-c \psi} d V$ is equal to $e^{-c \varphi}$ times the Lebesgue measure up to a strictly positive bounded function.

\subsection{Log canonical thresholds on group compactifications}

Let $X$ be a Fano compactification of $G$. Let $L$ be an ample linearized line bundle on $X$. Using Proposition 5.4, we reduce the computation of log canonical thresholds to integrability conditions on the potentials of metrics with respect to a smooth volume form. Since volume forms do not put weight on the (codimension one) boundary $X \backslash G$, we will restrict ourselves to integrability conditions on $G$. We want to use, in addition, the $K A K$-integration formula, that we recall here.

Proposition 5.5 ([Kna02, Proposition 5.28]). Let $d g$ denote a Haar measure on $G$ and $d x$ the Lebesgue measure on $\mathfrak{a}$, normalized by the lattice of one-parameter subgroups $N$. Then there exists a constant $C>0$ such that for any $K \times K$-invariant, $d g$-integrable function $f$ on $G$,

$$
\int_{G} f(g) d g=C \int_{\mathfrak{a}^{+}} J(x) f(\exp (x)) d x,
$$




\section{LOG CANONICAL THRESHOLDS ON GROUP COMPACTIFICATIONS}

where

$$
J(x)=\prod_{\alpha \in \Phi^{+}} \sinh (\alpha(x))^{2} .
$$

Let us now derive an integrability criterion with respect to $J$.

\subsection{Integrability criterion}

5.3.1 Integrability criterion on a cone. We use the following proposition, obtained by Guenancia in [Gue12]. It is an analytic proof and generalization of the computation by Howald of the log canonical thresholds of monomial ideals. The statement given here is slightly different from the statement in [Gue12], but is in fact equivalent (see [Del15a] for details).

Proposition 5.6 ([Gue12, Proposition 1.9]). Let $f$ be a convex function on a. Assume that $\sigma$ is a smooth polyhedral cone in $\mathfrak{a}=N \otimes \mathbb{R}$. Then $e^{-f}$ is integrable on a translate (equivalently, on all translates) of $\sigma$ if and only if 0 is in the interior of the Newton body of $f$, that is, $0 \in \operatorname{Int}\left(N_{\sigma}(f)\right)$.

5.3.2 Integrability with respect to $J$. The half sum of the positive roots of $\Phi$ is denoted by $\rho$. We want to prove the following integrability criterion with respect to the measure $J(x) d x$.

Proposition 5.7. Assume $\mathfrak{a}^{+}=\bigcup_{i} \sigma_{i}$, where each $\sigma_{i}$ is a smooth polyhedral cone of full dimension $r$. Let $l$ be a function on $\mathfrak{a}$, convex on each cone $\sigma_{i}$. Then

$$
\int_{\mathfrak{a}^{+}} e^{-l(x)} J(x) d x<+\infty
$$

if and only if $4 \rho \in \operatorname{Int}\left(N_{\mathfrak{a}^{+}}(l)\right)$.

Lemma 5.8. Let $\sigma$ be a smooth full-dimensional polyhedral cone in $\mathfrak{a}^{+}$, and let $l$ be a convex function on $\mathfrak{a}$; then the following are equivalent:

- $\int_{\sigma} e^{-l(x)} J(x) d x<\infty$.

- $\int_{\sigma} e^{-l(x)+4 \rho(x)} d x<\infty$.

- $4 \rho \in \operatorname{Int}\left(N_{\sigma}(l)\right)$.

Proof. Writing

$$
\sinh (\alpha(x))=\frac{e^{\alpha(x)}-e^{-\alpha(x)}}{2}=\frac{1}{2} e^{\alpha(x)}\left(1-e^{-2 \alpha(x)}\right)
$$

we get

$$
J(x)=\frac{1}{2^{2 \operatorname{Card}\left(\Phi^{+}\right)}} e^{2 \sum_{\alpha \in \Phi^{+}} \alpha(x)} \prod_{\alpha \in \Phi^{+}}\left(1-e^{-2 \alpha(x)}\right)^{2} .
$$

For any $x \in \mathfrak{a}^{+}$and $\alpha \in \Phi^{+}$, we have $\alpha(x)>0$, so $0 \leqslant e^{-2 \alpha(x)}<1$. This implies

$$
0<\prod_{\alpha \in \Phi^{+}}\left(1-e^{-2 \alpha(x)}\right)^{2} \leqslant 1
$$

so

$$
0<J(x) \leqslant \frac{1}{2^{2 \operatorname{Card}\left(\Phi^{+}\right)}} e^{4 \rho(x)} .
$$

This first inequality allows us to say that if $\int_{\sigma} e^{-l(x)+4 \rho(x)} d x<\infty$, then

$$
\int_{\sigma} e^{-l(x)} J(x) d x<\infty
$$




\section{T. DELCROIX}

Let us now prove the converse. Choose a point $\gamma$ in the interior of $\sigma$. Assume that $e^{-l+4 \rho}$ is not integrable on $\sigma$. Then by the usual integrability criterion (Proposition 5.6), $e^{-l+4 \rho}$ is also non-integrable on $\gamma+\sigma$.

But now, for $x \in \gamma+\mathfrak{a}^{+}$and $\alpha \in \Phi^{+}$, we have $\alpha(x) \geqslant c=\min _{\beta \in \Phi^{+}} \beta(\gamma)>0$, so $0 \leqslant e^{-2 \alpha(x)} \leqslant$ $e^{-2 c}<1$, and this implies

$$
\left(\frac{1-e^{-2 c}}{2}\right)^{2 \operatorname{Card}\left(\Phi^{+}\right)} e^{4 \rho(x)} \leqslant J(x) \leqslant \frac{1}{2^{2 \operatorname{Card}\left(\Phi^{+}\right)}} e^{4 \rho(x)} .
$$

This gives

$$
\int_{\sigma} e^{-l(x)} J(x) d x \geqslant \int_{\gamma+\sigma} e^{-l(x)} J(x) d x \geqslant \int_{\gamma+\sigma} e^{-l+4 \rho} d x \geqslant \infty .
$$

We have shown the equivalence of the first two points in the lemma. By Proposition 5.6 the second point is also equivalent to

$$
0 \in \operatorname{Int}\left(N_{\sigma}(l-4 \rho)\right)=-4 \rho+\operatorname{Int}\left(N_{\sigma}(l)\right) .
$$

We conclude the proof by moving $4 \rho$ to the left.

Now, we can prove the proposition, just by gluing the parts.

Proof of Proposition 5.7. Just remark that since the function $e^{-l(x)} J(x)$ is positive and the cones are full-dimensional, $\int_{\mathfrak{a}^{+}} e^{-l(x)} J(x) d x<+\infty$ if and only if $\int_{\sigma_{i}} e^{-l(x)} J(x) d x<+\infty$ for all $i$.

For each of these integrals we can use the lemma, so the necessary and sufficient condition becomes $4 \rho \in \operatorname{Int}\left(N_{\sigma_{i}}(l)\right)$ for all $i$ or, equivalently, $4 \rho \in \operatorname{Int}\left(\bigcap_{i} N_{\sigma_{i}}(l)\right)$.

To conclude, observe that $N_{\mathfrak{a}^{+}}(l)=\bigcap_{i} N_{\sigma_{i}}(l)$ by Proposition 4.3.

\subsection{Log canonical thresholds in terms of Newton bodies}

Let $X$ be a Fano compactification of $G$. Let $L$ be a linearized ample line bundle on $X$ whose associated polytope is $P$. Denote by $Q$ the polytope associated with the anticanonical bundle $-K_{X}$. Let also $H$ denote the convex hull of all images of $2 \rho$ by the Weyl group $W$.

We want to prove the following.

TheOREm 5.9. Let $h$ be a $K \times K$-invariant hermitian metric with non-negative curvature on $L$; then

$$
\operatorname{lct}(h)=\sup \{c>0 ; 2 H+2 c P \subset c N(h)+2 Q\} .
$$

We first introduce some notation. Let us fix a left $G$-equivariant trivialization $s_{0}$ of $L$ on $G$ and a left $G$-equivariant trivialization $s_{1}$ of $-K_{X}$ on $G$.

Let $u$ be the convex potential of $h$ with respect to the section $s_{0}$. Let $u_{0}$ be the support function of $P$ and $h_{0}$ the corresponding metric. It has locally bounded potentials. Denote by $\psi$ the potential of $h$ with respect to $h_{0}$.

Since $X$ is Fano, we can choose a smooth metric $h_{1}$ on $-K_{X}$ with positive curvature; let $u_{1}$ be its convex potential with respect to $s_{1}$. This choice determines a smooth volume form on $X$, which on $G$ can be written as

$d V=e^{-u_{1}} d g$

where $d g$ is the Haar measure $s_{1}^{-1} \wedge \overline{s_{1}^{-1}}$. 


\section{LOG CANONICAL THRESHOLDS ON GROUP COMPACTIFICATIONS}

Remark 5.10. In particular, the integral of this volume form is finite, so by applying the $K A K$ integration formula, we see that

$$
\int_{\mathfrak{a}^{+}} e^{-u_{1}} J d x<\infty
$$

By Proposition 5.7, this implies

$$
4 \rho \in \operatorname{Int}\left(N\left(h_{1}\right)\right)=\operatorname{Int}(2 Q) .
$$

Another way to say this is $H \subset \operatorname{Int}(Q)$.

Proof of Theorem 5.9. Using Proposition 5.4, then restricting to the dense orbit, we get

$$
\begin{aligned}
\operatorname{lct}(h) & =\sup \left\{c>0 ; \int_{X} e^{-c \psi} d V<\infty\right\} \\
& =\sup \left\{c>0 ; \int_{G} e^{-c \psi} d V<\infty\right\} .
\end{aligned}
$$

Since $\psi(\exp (x))=u(x)-u_{0}(x)$, we can now use the $K A K$-integration formula to write

$$
\operatorname{lct}(h)=\sup \left\{c>0 ; \int_{\mathfrak{a}^{+}} e^{-c\left(u-u_{0}\right)} e^{-u_{1}} J d x<\infty\right\} .
$$

Then Proposition 5.7 gives

$$
\begin{aligned}
\operatorname{lct}(h) & =\sup \left\{c>0 ; 4 \rho \in \operatorname{Int}\left(N_{\mathfrak{a}^{+}}\left(c u-c u_{0}+u_{1}\right)\right)\right\} \\
& =\sup \left\{c>0 ; 4 \rho \in N_{\mathfrak{a}^{+}}\left(c u-c u_{0}+u_{1}\right)\right\} .
\end{aligned}
$$

Let $\sigma_{i}$ be the cones of full dimension in the fan subdivision of $\mathfrak{a}^{+}$corresponding to $X$ (induced by the fan subdivision of $\mathfrak{a}$ associated with the toric subvariety $Z$ ). Then $u_{0}$ is linear on each $-\sigma_{i}$. We write $u_{0}^{i}$ for the corresponding element of $\mathfrak{a}^{*}$.

We have

$$
\begin{aligned}
\operatorname{lct}(h) & =\sup \left\{c>0 ; \forall i, 4 \rho \in N_{-\sigma_{i}}\left(c u-c u_{0}+u_{1}\right)\right\} \\
& =\sup \left\{c>0 ; \forall i, 4 \rho+c u_{0}^{i} \in N_{-\sigma_{i}}\left(c u+u_{1}\right)\right\} .
\end{aligned}
$$

Recall from Example 4.4 that $P=N_{\mathfrak{a}}\left(u_{0}\right) \subset u_{0}^{i}+\sigma_{i}^{\vee}$, so that

$$
\begin{aligned}
\operatorname{lct}(h) & =\sup \left\{c>0 ; \forall i, 4 \rho+c P \in N_{-\sigma_{i}}\left(c u+u_{1}\right)\right\} \\
& =\sup \left\{c>0 ; 4 \rho+c P \in N_{\mathfrak{a}^{+}}\left(c u+u_{1}\right)\right\} \\
& =\sup \left\{c>0 ; 2 H+2 c P \subset N_{\mathfrak{a}}\left(c u+u_{1}\right)\right\}
\end{aligned}
$$

by $W$-invariance.

To conclude it remains to remark that both $u$ and $u_{1}$ are convex, so by Proposition 4.8,

$$
N_{\mathfrak{a}}\left(c u+u_{1}\right)=c N_{\mathfrak{a}}(u)+N_{\mathfrak{a}}\left(u_{1}\right)=c N(h)+2 Q .
$$

\section{Alpha invariants}

In this section, we obtain an expression for the $\alpha$-invariant of a polarized group compactification in terms of its polytope. We first give the result for general reductive group compactifications, then see how it simplifies when the group is semisimple. We then discuss some examples and how some additional symmetries can be taken into account for reductive group compactifications. 


\section{T. DELCROIX}

\subsection{The general formula}

Definition 6.1. Let $X$ be a compact complex manifold, $K$ a compact subgroup of the automorphisms group of $X$, and $L$ a $K$-linearized line bundle on $X$. The alpha invariant of $L$ relative to the group $K$, denoted by $\alpha_{K}(L)$, is the infimum of the log canonical thesholds of all $K$-invariant singular hermitian metrics on $L$ with non-negative curvature.

Let $P$ and $Q$ be two convex bodies in $\mathfrak{a}^{*}$. Recall the definition of the Minkowski difference:

$$
Q \ominus P=\{x \mid x+P \subset Q\} .
$$

Another expression of the Minkowski difference is the following, which shows that it is convex if $Q$ is convex:

$$
Q \ominus P=\bigcap_{p \in P}(-p+Q) .
$$

If $P_{1}, P_{2}$ and $Q$ are three convex bodies, then $P_{1}+Q \subset P_{2}$ if and only if $P_{1} \subset P_{2} \ominus Q$.

We can now state our main result.

Theorem 6.2. Let $(X, L)$ be a polarized compactification of $G$ and $P:=P(X, L)$. Assume furthermore that $X$ is Fano, and let $Q:=P\left(X,-K_{X}\right)$. Then

$$
\alpha_{K \times K}(L)=\sup \left\{c>0 ; c\left(P+\left(-P^{W}\right)\right) \subset Q \ominus H\right\},
$$

where $P^{W}$ denotes the subset of $W$-invariant points of $P$.

Proof. Let $h$ be any $K \times K$-invariant metric on $L$ with non-negative curvature. The Newton body of $h$ is convex and $W$-stable. In particular, it contains a $W$-invariant point $p$, for example the barycenter of the orbit of any point in $N(h)$.

Denote by $h_{p}$ the $K \times K$-invariant metric on $L$ with non-negative curvature whose convex potential is the function $x \mapsto p(x)$. Then $\{p\}=N\left(h_{p}\right) \subset N(h)$, so by the expression of the log canonical thresholds from Theorem 5.9, we have $\operatorname{lct}(h) \geqslant \operatorname{lct}\left(h_{p}\right)$.

Since all such $h_{p}$ for $p \in 2 P^{W}$ define a singular hermitian metric with non-negative curvature, this remark allows us to write the alpha invariant as

$$
\alpha_{K \times K}(L)=\inf _{p \in 2 P W} \operatorname{lct}\left(h_{p}\right) .
$$

Now, from the expression of the log canonical threshold, we get

$$
\begin{aligned}
\operatorname{lct}\left(h_{p}\right) & =\sup \left\{c>0 ; 2 H+2 c P \subset c N\left(h_{p}\right)+2 Q\right\} \\
& =\sup \{c>0 ;-c p+2 c P \subset 2 Q \ominus 2 H\} .
\end{aligned}
$$

Then, the expression of the alpha invariant further simplifies as

$$
\begin{aligned}
\alpha_{K \times K}(L) & =\inf _{p \in 2 P W} \sup \{c>0 ;-c p+2 c P \subset 2 Q \ominus 2 H\} \\
& =\sup \left\{c>0 ; \forall p \in 2 P^{W},-c p+2 c P \subset 2 Q \ominus 2 H\right\} \\
& =\sup \left\{c>0 ; 2 c P+\left(-2 c P^{W}\right) \subset 2 Q \ominus 2 H\right\} .
\end{aligned}
$$

Dividing by 2 yields

$$
=\sup \left\{c>0 ; c\left(P+\left(-P^{W}\right)\right) \subset Q \ominus H\right\},
$$

which is the expression in the statement of the theorem. 


\section{LOG CANONICAL THRESHOLDS ON GROUP COMPACTIFICATIONS}

Remark 6.3. In the toric case, we recover our previous computation [Del15a]:

$$
\alpha_{\left(\mathbb{S}^{1}\right)^{n}}(L)=\sup \{c>0 ; c(P+(-P)) \subset Q\} .
$$

\subsection{The semisimple case}

The alpha invariant of an ample line bundle on a Fano compactification of a semisimple group can easily be expressed in terms of the polytope associated with $L$ as an inradius between two convex bodies.

Definition 6.4. The inradius of $Q$ with respect to $P$ is the number

$$
\operatorname{inr}(P, Q):=\sup \{c \geqslant 0 \mid \exists x, x+c P \subset Q\} .
$$

Corollary 6.5. Assume that $G$ is a semisimple group. Then

$$
\alpha_{K \times K}(L)=\operatorname{inr}(P, Q \ominus H) .
$$

Proof. If $G$ is semisimple, we have $P^{W}=\{0\}$. In fact, the metric $h_{0}$ whose convex potential is the zero function satisfies

$$
\alpha_{K \times K}(L)=\operatorname{lct}\left(h_{0}\right)=\sup \{c>0 ; c P \subset Q \ominus H\} .
$$

This is equal to the inradius $\operatorname{inr}(P, Q \ominus H)$. Indeed, one inequality is trivial: $\operatorname{inr}(P, Q \ominus H) \geqslant$ $\alpha_{K \times K}(L)$. Conversely, assume $c \leqslant \operatorname{inr}(P, Q \ominus H)$, that is, there exists an $x \in \mathfrak{a}^{*}$ such that

$$
x+c P \subset Q \ominus H .
$$

Then, since $P$ and $Q \ominus H$ are stable under $W$-action, we also have

$$
\forall w \in W, \quad w \cdot x+c P \subset Q \ominus H .
$$

Convexity and the fact that the barycenter of the $W$-orbit of $x$ is 0 imply that $c P \subset Q \ominus H$, so $c \leqslant \alpha_{K \times K}(L)$. We have thus proved the other inequality $\operatorname{inr}(P, Q \ominus H) \leqslant \alpha_{K \times K}(L)$.

Remark 6.6. In the case of reductive groups, the alpha invariant is not an inradius, but we can bound it from above by an inradius:

$$
\alpha_{K \times K}(L) \leqslant \operatorname{inr}\left(P+(-P)^{W}, Q \ominus H\right) .
$$

\subsection{Additional symmetries}

If the polytopes $P$ and $Q$ admit additional common symmetries, then the value of the alpha invariant can be improved. Indeed, the symmetries of $Q$ translate to a finite subgroup $O$ of the automorphisms group of the variety $X$, and if $P$ is stable under these symmetries, then it is linearized by $O$. We can thus consider the alpha invariant with respect to the bigger group generated by $K \times K$ and $O$, that we denote $K_{O}$.

We then have, adapting the proof of Theorem 6.2,

$$
\alpha_{K_{O}}(L)=\sup \left\{c>0 ; c\left(P+\left(-P^{\langle W, O\rangle}\right)\right) \subset Q \ominus H\right\} .
$$

In particular, if the only fixed point under $\langle W, O\rangle$ is the origin, then just as in the semisimple case, we get

$$
\alpha_{K_{O}}(L)=\operatorname{inr}(P, Q \ominus H) .
$$




\section{T. DELCROIX}

\subsection{Examples}

Let us compute the $\alpha$-invariant of the anticanonical line bundle for some wonderful compactifications of semisimple groups. First, remark that in this case we have $P=Q$ and can rewrite the expression of the invariant as

$$
\alpha_{K \times K}\left(X,-K_{X}\right)=\sup \{c>0 ; H \subset(1-c) Q\}
$$

or, by $W$-invariance,

$$
\alpha_{K \times K}\left(X,-K_{X}\right)=\sup \left\{c>0 ; 2 \rho \in(1-c) Q^{+}\right\} .
$$

Remark 6.7. It is interesting to notice that this quantity appeared in the determination of some volume asymptotics of height balls in analytic varieties over local fields and in adelic points of algebraic varieties over local fields by Chambert-Loir and Tschinkel. If $\sigma$ denotes the quantity the authors compute in the examples of compactifications of semisimple groups [CLT10, Section 5.3], we have $\sigma=1-\alpha_{K \times K}\left(X,-K_{X}\right)$ if the considered polytope is the anticanonical polytope of a Fano compactification. A basic interpretation is that, in the end, their computation in this special case reduces to a computation of the log canonical threshold of a metric on the anticanonical line bundle with constant convex potential. It would, however, be interesting to investigate the possible deeper relation between the two quantities.

For wonderful compactifications of semisimple adjoint groups, the polytope of the anticanonical line bundle $Q$ is determined by the root system. Indeed, recall that it is the convex hull of the images by $W$ of the weight $2 \rho+\sum_{i=1}^{r} \alpha_{i}$, where the $\alpha_{i}$ are the simple roots of $\Phi^{+}$.

In particular, when $G=\left(\mathrm{PSL}^{2}(\mathbb{C})\right)^{n}$, for any $n \geqslant 1$, the simple roots are the same as the positive roots, so $Q=2 H$.

Corollary 6.8. Let $X$ be the wonderful compactification of $\left(\mathrm{PSL}_{2}(\mathbb{C})\right)^{n}$; then

$$
\alpha_{K \times K}\left(-K_{X}\right)=\frac{1}{2}
$$

Proof. Applying Corollary 6.5 gives $\alpha_{K \times K}\left(-K_{X}\right)=\operatorname{inr}(2 H, H)=\frac{1}{2}$.

More generally, for type $A_{n}$, choosing an appropriate ordering of the simple roots $\alpha_{1}, \ldots, \alpha_{n}$, we can write the positive roots as

$$
\alpha_{i}+\alpha_{i+1}+\cdots+\alpha_{j}
$$

for all pairs $(i, j)$ with $1 \leqslant i \leqslant j \leqslant n$. We then see that the coefficient of $\alpha_{k}$ in the sum of positive roots $\sum_{l=1}^{n} \alpha_{l}$ is equal to the cardinality of the set $\{(i, j) ; 1 \leqslant i \leqslant k \leqslant j \leqslant n\}$. This is $k(n-k+1)$. Adding the sum of simple roots, we see that the $k$ th coordinate of the vertex defining the polytope of the wonderful compactification of $\mathrm{PSL}_{n+1}(\mathbb{C})$ in the basis of simple roots is $1+k(n-k+1)$.

Then, from our result, the alpha invariant is easily seen to be the maximum of all $c>0$ such that for each $k$, we have $c(1+k(n-k+1)) \leqslant 1$. We deduce the following value for the alpha invariant.

Corollary 6.9. Let $X$ be the wonderful compactification of $\mathrm{PSL}_{n+1}(\mathbb{C})$; then

$$
\alpha_{K \times K}\left(-K_{X}\right)=\frac{1}{1+\left\lceil\frac{n}{2}\right\rceil\left(\left\lfloor\frac{n}{2}\right\rfloor+1\right)} .
$$




\section{LOG CANONICAL THRESHOLDS ON GROUP COMPACTIFICATIONS}

\section{REFERENCES}

AB04 V. Alexeev and M. Brion, Stable reductive varieties. II. Projective case, Adv. Math. 184 (2004), no. 2, 380-408; https://doi.org/10.1016/S0001-8708(03)00164-6.

AL92 H. Azad and J.-J. Loeb, Plurisubharmonic functions and Kählerian metrics on complexification of symmetric spaces, Indag. Math. (N.S.) 3 (1992), no. 4, 365-375; https://doi .org/10.1016/ 0019-3577 (92) 90017-F.

Amb14 F. Ambro, Variation of log canonical thresholds in linear systems, 2014, arXiv:1411.2770.

BK05 M. Brion and S. Kumar, Frobenius splitting methods in geometry and representation theory, Progress in Mathematics, vol. 231 (Birkhäuser Boston, Inc., Boston, MA, 2005); https://doi. org/10.1007/b137486.

Bri07 M. Brion, The total coordinate ring of a wonderful variety, J. Algebra 313 (2007), no. 1, 61-99; https://doi.org/10.1016/j.jalgebra.2006.12.022.

CLT10 A. Chambert-Loir and Y. Tschinkel, Igusa integrals and volume asymptotics in analytic and adelic geometry, Confluentes Math. 2 (2010), no. 3, 351-429; https://doi.org/10.1142/ S1793744210000223.

DCP83 C. De Concini and C. Procesi, Complete symmetric varieties, Invariant Theory (Montecatini, 1982), Lecture Notes in Math., vol. 996 (Springer, Berlin, 1983), 1-44; https://doi.org/10. $1007 / \mathrm{BFb} 0063234$.

Del15a T. Delcroix, Alpha-invariant of toric line bundles, Ann. Polon. Math. 114 (2015), no. 1, 13-27; https://doi.org/10.4064/ap114-1-2.

Del15b _ Métriques de Kähler-Einstein sur les compactifications de groupes, Ph.D. thesis, Université Grenoble Alpes, 2015, available at https://tel.archives-ouvertes.fr/tel-01286292.

Del17_ Kähler-Einstein metrics on group compactifications, Geom. Funct. Anal., electronically published on 30 January 2017, https://doi.org/10.1007/s00039-017-0394-y, to appear in print.

Ful93 W. Fulton, Introduction to toric varieties, Ann. of Math. Stud., vol. 131 (Princeton Univ. Press, Princeton, NJ, 1993).

Gue12 H. Guenancia, Toric plurisubharmonic functions and analytic adjoint ideal sheaves, Math. Z. 271 (2012), no. 3-4, 1011-1035; https://doi.org/10.1007/s00209-011-0900-0.

Kna02 A. W. Knapp, Lie groups beyond an introduction, 2nd ed., Progr. Math., vol. 140 (Birkhäuser Boston, Inc., Boston, MA, 2002); https://doi.org/10.1007/978-1-4757-2453-0.

LSY15 H. Li, Y. Shi and Y. Yao, A criterion for the properness of the $K$-energy in a general Kähler class, Math. Ann. 361 (2015), no. 1-2, 135-156; https://doi.org/10.1007/ s00208-014-1073-z.

Mai00 V. Maillot, Géométrie d'Arakelov des variétés toriques et fibrés en droites intégrables, Mém. Soc. Math. Fr. (N.S.) (2000), no. 80; http://www.numdam.org/item?id=MSMF_2000_2_80__R3_0.

Oda88 T. Oda, Convex bodies and algebraic geometry. an introduction to the theory of toric varieties, Ergeb. Math. Grenzgeb. (3), vol. 15 (Springer-Verlag, Berlin, 1988).

Per14 N. Perrin, On the geometry of spherical varieties, Transform. Groups 19 (2014), no. 1, 171-223; https://doi.org/10.1007/s00031-014-9254-0.

Roc97 R. T. Rockafellar, Convex analysis, Princeton Landmarks in Math. (Princeton Univ. Press, Princeton, NJ, 1997).

Son05 J. Song, The $\alpha$-invariant on toric Fano manifolds, Amer. J. Math. 127 (2005), no. 6, 1247-1259; https://doi.org/10.1353/ajm.2005.0043.

STBT07 J. Shalika, R. Takloo-Bighash and Y. Tschinkel, Rational points on compactifications of semisimple groups, J. Amer. Math. Soc. 20 (2007), no. 4, 1135-1186; https://doi.org/10.1090/ S0894-0347-07-00572-3. 


\section{T. DELCROIX}

Tia87 G. Tian, On Kähler-Einstein metrics on certain Kähler manifolds with $C_{1}(M)>0$, Invent. Math. 89 (1987), no. 2, 225-246; https://doi.org/10.1007/BF01389077.

Tim11 D. A. Timashev, Homogeneous spaces and equivariant embeddings, Encyclopaedia of Math. Sci., vol. 138 (Springer, Heidelberg, 2011); https://doi .org/10.1007/978-3-642-18399-7.

Thibaut Delcroix thibaut.delcroix@ujf-grenoble.fr

Université Grenoble Alpes, IF, 38000 Grenoble, France

CNRS, IF, 38000 Grenoble, France

Current address: Département de mathématiques et applications, École normale supérieure, 45 rue d'Ulm, 75230 Paris Cedex 05, France 